\title{
Antibacterial Spectrum and Susceptibility of Bacterial Pathogens Causing Diarrheal Illnesses: Cross Sectional Study of Patients Visiting Health Facility in Lake Victoria Region - Kenya
}

\author{
Makwaga Olipher ${ }^{\mathrm{a}}$, Muyodi Johnstone ${ }^{\mathrm{a}}$, Muthami Anne ${ }^{\mathrm{b}}$, Malela Joseph ${ }^{\mathrm{a}}$, Mokaya Tomª, Matilu Mwau \\ ${ }^{a}$ Center for Infectious and Parasitic Diseases Control Research, Kenya Medical Research Institute \\ ${ }^{\mathrm{b}}$ Masinde Muliro University of Science and Technology \\ *Correspondence to Olipher Makwaga (oliphermakwaga@gmail.com)
}

\begin{abstract}
Diarrheal illness has been studied extensively among children under five years, however little information is known among population over five years. This study determined the spectrum and antibiotic susceptibility of bacterial pathogens causing diarrhea in above five-year-old patients in a health facility along Lake Victoria region. In a cross-sectional study, 400 patients aged five years and above with diarrhea visiting Port Victoria health facility along Lake Victoria region were recruited in this study from 2013 to 2016. Stool samples were collected and cultured following standard microbiological procedures. Bacterial agents were isolated, identified and subjected to antibiotic susceptibility test. A total of 317 organisms (283 bacteria, 31 parasites and 3 others) were identified from $303(76 \%)$ patients. Most frequently isolated pathogens being Non O1 Vibrio cholera 98(30.9\%), Salmonella species 97(30.6\%), Shigella species 47(14.8.0\%) and Aeromonas species $38(12.0 \%)$. Non O1 Vibrio cholerae isolates were less sensitive to tetracycline, ampicillin and nalidixic acid. Salmonella species were less sensitive to ampicillin but sensitive to nalidixic acid. Shigella species isolates were more sensitive to chloramphenicol, cipplofloxin and nalidixic acid. Similar trend was observed for Aeromonas species isolates. The susceptibilities expressed were fairly good. However, some of the resistances shown pose a threat to public health, especially in unexposed populations. With the observed trends in this study, bacterial species have responded differently to antibiotics. It may be beneficial for clinicians to consider antibiotic susceptibility testing prior to management of patients to slow down resistance development.
\end{abstract}

Key words: Diarrheal illness, Bacterial pathogens, Antibiotic susceptibility, Vibrio cholerae, Salmonella

\section{INTRODUCTION}

1 iarrheal diseases are common world-wide and among the leading causes of morbidity and mortality in developing nations. World Health Organization (WHO) report showed that diarrheal disease was the fifth most common infection accounting to mortality of $3.7 \%$ (2.2 million) in 2004 ${ }^{1}$. In 2010, 1.4 million people, across all ages, died from diarrheal diseases, and the age-standardized mortality rate was 20.9 per 100,000 , which has fallen to $49 \%$ between by $2010^{2}$. Diarrhea is the global second leading cause of mortality, and nearly one in five children under the age of five die as a result of dehydration, weakened immunity or malnutrition associated with diarrhea ${ }^{3}$.
Mortality rates among children declined globally from 146 per 1,000 in 1970 to 79 per 1,000 in $2003^{4}$. However, the situation in Africa is strikingly different compared with other regions of the world. African region shows the smallest reductions in mortality rates and the most marked slowing down trend ${ }^{5}$. Diarrheal illness is the third leading cause of morbidity and mortality in children younger than 5 years of age in Africa and were responsible for 30 million cases of severe diarrhea and 330,000 deaths in $2015^{6}$. Diarrhea remains a major public health problem in East African, for instance, the prevalence of diarrhea among children less than five years ranged from 11-54\% with an average of $27 \%$ in Kenya, Ethiopia, and Somalia from 2012 to $2017^{7}$. Between 
2001 and 2003, diarrhea was responsible for $11.2 \%$ hospitalized cases in Kenya. Fatality rate was $8.0 \%$ with more adults than children ${ }^{8}$. Diarrhea is the third most common pediatric diagnosis in rural western Kenya and particularly among adults with HIV infection ${ }^{9,10,11}$. Although extensive diarrheal studies have been carried out in children below the age of five years, paucity exists in individuals older than this age. Therefore, this study sought to determine bacterial agents causing diarrhea in individuals older than five years and also determine the antibiotic susceptibility test using commonly available antibiotics.

Diarrhea is the passage of three or more loose stools per day by an individual ${ }^{12}$. It is usually a symptom of gastrointestinal infection. Diarrhea diseases due to infection constitute a major burden of disease ${ }^{12}$. Systematic analysis for the Global Burden of Disease (GBD) indicated that there are various aetiologies for diarrhea in the cause list for $2010^{2}$. Diarrhea can be caused by bacteria, viruses or parasites ${ }^{13,14,15}$. Among the major diarrheal bacteria include Vibrio species, Salmonella paratyphi, Shigella, Enterotoxigenic E. coli $^{16,17,18}$. In fact, some constitute acute infectious diarrhea ${ }^{19}$. Cholera remains an important cause of illness in many developing countries and has been estimated to result in more than 120,000 deaths each year ${ }^{20}$. The most readily recognized bacterial agents of gastroenteritis in industrialized countries are Salmonella spp and Campylobacter $\mathrm{Spp}^{13,21,22}$. Bacillary dysentery due to either Shigella dysenteriae type 1 or Entoamoeba contributes to a large degree of bloody diarrhea illness based on reports from Kenya, Egypt, Ethiopia and Nicaragua ${ }^{23,24,25}$. The various Shigella species can cause diarrhea or the syndrome known as bacillary dysentery. Severe acute diarrhea is more common in travelers and older people. Travelers are exposed to drinking contaminated water and eating contaminated food prepared by other people at their destinations whereas older people have lower immunity to fight against pathogens causing diarrhea ${ }^{26}$. Globally, Shigella was the second leading cause of diarrheal mortality in 2016, accounting for 212438 deaths among all ages and responsible for 63713 deaths among children younger than 5 years ${ }^{27}$.

World-wide, the etiology of bacteria causing diarrhea appears to differ depending on the geographical area. For example, a report from Spain indicated that Campylobacter spp. and Salmonella spp. were the primary bacterial pathogens, accounting for $22.2 \%$ and $16.4 \%$ of cases of acute diarrhea in children respectively ${ }^{28}$. In Ecuador, Shigella spp. and Campylobacter jejuni were reported to be the main etiological causes of diarrhea ${ }^{29}$. Whereas in Turkey, Salmonella spp. (25.6\%) and $C$. jejuni $(18.3 \%)$ were the main causes of acute gastroenteritis in children ${ }^{30}$. In developing countries, Shigella, non- typhoidal Salmonella (NTS), Campylobacter, and Yersinia are the major enteropathogens causing diarrhea across all ages $^{31}$. 22.2\% diarrheal cases were caused by Aeromonas species in Pakistan and Bangladesh between 2009 and 201232. A Case-Control Study in Ifakara, Tanzania reported $47.8 \%$ children with diarrhea caused by enteropathogen of which Shigella species was the most prevalent ${ }^{33}$.

As much as in Kenya Vibrio species has been isolated from stool of diarrheal patients during outbreaks ${ }^{9,10,11}$ a prevalence of $18.8 \%$ and fatality rate of $2.5 \%$ was reported among diarrheal patients across all the ages ${ }^{9}$. Salmonella species have been isolated in studies carried out in Kenya $34,35,36,37,38,39,40,41,42,43$. However, a prevalence of 14 was reported among children of less than 12 years of age ${ }^{44}$. In a study carried out in Kenya on patients with sporadic bloody diarrhea, Shigella species was the most prevalent pathogen affecting $44 \%$ of the studied population followed by Salmonella species with $17 \% \%^{45}$. In two population-based studies, $22.3 \%$ and $23 \%$ incidences of shigellosis in Nairobi, Kenya were reported ${ }^{46,47}$. Aeromonas associated diarrheal study in Kenya reported $0 \%$ isolation of this pathogen from less than 5 years old children with diarrhea ${ }^{32}$. However Aeromonas Hydrophila was isolated from River Njoro in Kenya in $2012^{48}$. Another study in India reported $17.7 \%$ prevalence of Aeromonas hydrophila from patients with diarrhea $^{49}$. The assumption was, there could be other more bacterial causing diarrheal illness. Therefore, this study was designed with an aim of determining if there could be other more bacterial pathogens causing diarrhea in addition to the ones that are documented.

In Africa, including Kenya, the diagnosis of diarrheal illness is based on clinical and laboratory investigations of pathogens causing diarrhea. Clinically, the doctor investigates the patients based on the presenting syndromes i.e. acute watery stool, three or more episodes of diarrhea per day or bloody diarrhea by examining the rectum. Laboratory investigations involve two aspects i.e. the use of simple microscopy technique on a stool sample to be able to identify protozoa or helminthes causing diarrhea. The second laboratory diagnosis is microbiological culture of stool sample to be able to identify bacteria causing diarrhea.

In treatment of different bacterial diseases, antibiotics such as tetracycline, doxycycline, erythromycin and streptomycin are generally used ${ }^{50}$. Antimicrobial resistance is one of the most important public health problems that directly relates to disease management and control ${ }^{51}$. Resistance to antibiotics is an increasingly common problem in human medicine. Its management is the subject of urgent debate. Efforts to reduce this resistance are based on the assumption 
that it is maintained in bacterial populations as a result of exposure to antibiotics, and restricting the use of antibiotics $^{52}$. With few exceptions, the major classes of antibiotics used to manage infections with enteric bacteria include the beta-lactams (penicillin derivatives), carbapenems, the fluoroquinolones, the aminoglycosides, and TMP-SMZ. Because most Enterobacter species are either very resistant to many agents or can develop resistance during antimicrobial therapy, the choice of appropriate antimicrobial agents is complicated ${ }^{53,54}$. In Kenya there are diverse commonly prescribed antibiotics for treating infections caused by enteric bacteria. These include, tetracycline, gentamicin, doxycycline, norfloxacin, ciprofloxacin, streptomycin, chloramphenical, cotrimoxazole, Nalidixic acid, Ampicilin, cefotoxime e.t.c ${ }^{47}$. Antibiotics such as tetracycline, doxycycline, norfloxacin, ciprofloxacin and streptomycin are used with adjunct in rehydration therapy and are critical in the treatment of septicemia patient ${ }^{50,55,56}$. Resistance to many of these drugs in general Vibrio pathogens have been reported in various African countries including Kenya ${ }^{51,57,58,59,60,61,62}$. Antibiotic resistance of bacterial pathogens isolated from childhood diarrhea in four provinces of Kenya reported resistance of $100 \%$ of Vibrio pathogens to Nalidixic acid and chloramphenicol; sensitivity of $100 \%$ to ciprofloxacin and tetracycline and resistance of $50 \%$ to ampicillin ${ }^{60}$.

Studies carried out for more than 10 years ago have reported resistance of Salmonella strains to commonly prescribed antibiotics in some parts of Kenya ${ }^{34,35,36,37,38,39,40,41}$. A study carried out in Kenya reported Salmonella level of resistance ranged from $44.4 \%$ to $75 \%$ for ampicillin; $22.2 \%$ to $50 \%$ for chloramphenicol; $33.3 \%$ to $66.7 \%$ for tetracycline; $0 \%$ to $25 \%$ for nalidixic acid and sensitivity of $100 \%$ to ciprofloxacin ${ }^{60}$. Treatment of shigellosis by appropriate antimicrobial agents has proven efficacious in shortening the duration of fever, diarrhea and toxaemia and apparently in reducing the risk of lethal complications as well ${ }^{63}$. The increase in the number of Shigella isolates resistant to most of the antibiotics (sulfonamides, tetracyclines, ampicillin, and trimethoprim-sulfamethoxazole (TMP-SMX) available in countries where the choice of treatment is limited ${ }^{34}$, represents an important health problem. The World Health Organization (WHO) recommends that all suspected cases of shigellosis based on clinical features be treated with effective antimicrobials (antibiotics) ${ }^{64}$. If Shigella dysenterea has been isolated, Beta-lactams (Ampicillin, amoxicillin, third-generation cephalosporins (cefixime, ceftriaxone), and pivmecillinam); Quinolones: (Nalidixic acid, ciprofloxacin, norfloxacin, and ofloxacin); Macrolides (Azithromycin) and others (sulfonamides, tetracycline, cotrimoxazole, and furazolidone) can be used for treatment. Resistance of this organism against antibiotics is has been reported in Kenya ${ }^{12}$. A study carried out among children in four provinces of Kenya reported sensitivity of $75 \%$ to $100 \%$ of Shigella pathogen to ciprofloxacin and nalidixic acid; $50 \%$ to $100 \%$ to chloramphenicol; $20 \%$ to $100 \%$ to tetracycline and resistance of $33.3 \%$ to $80 \%$ to ampicillin ${ }^{60}$.

\section{Rationale}

From the literature, diarrheal illness has been studied extensively among children under five years, however, little information is known among the population over five years. Furthermore, different levels of resistances and sensitivities of these organisms against commonly prescribed antibiotics have been reported in some parts of Kenya ${ }^{12,60,61,62}$. However, little information has been reported in the current study site. Therefore, this study was designed to determine etiological agents causing diarrheal illnesses among individuals of five years and above and determine antibiotic susceptibility profile of these enteric pathogen and advice the clinicians on the appropriate antibiotics to use for managing diarrheal patients.

\section{METHODS}

\section{Study design}

This was a cross sectional study involving patients presenting at Port Victoria hospital with three or more episodes of diarrhea per day during rainy season (April, May, June) and (August and September). A total of 100 samples were collected every year within these five months, 20 samples per months and an average of 1 sample per-day.

\section{Study site}

The study took place at Port Victoria Sub-county hospital (Figure 1) in Busia County, Kenya. This health facility borders Lake Victoria and located about $2 \mathrm{~km}$ away. Majority of residents are fisher folk from Luo and Luhyia communities. The hospital offers the following services: Antenatal, Antiretroviral Therapy, Basic comprehensive Emergency Obstetric Care, Caesarean, Curative In-patient Services, Curative Outpatient Services, Family Planning, Growth Monitoring and Promotion, HIV Counseling and Testing, Immunization, Integrated Management of Childhood Illnesses, Prevention of Mother to Child transmission of HIV, Radiology (e.g. X-ray, UltraScan, MRI, etc.), Tuberculosis Diagnosis, and Treatment, Laboratory and pharmaceutical services. Among the most diagnosed diseases are Malaria, HIV, diarrhea, pneumonia, tuberculosis etc. The climate of the area is warm and hot with two distinct seasons, dry and rainy. 


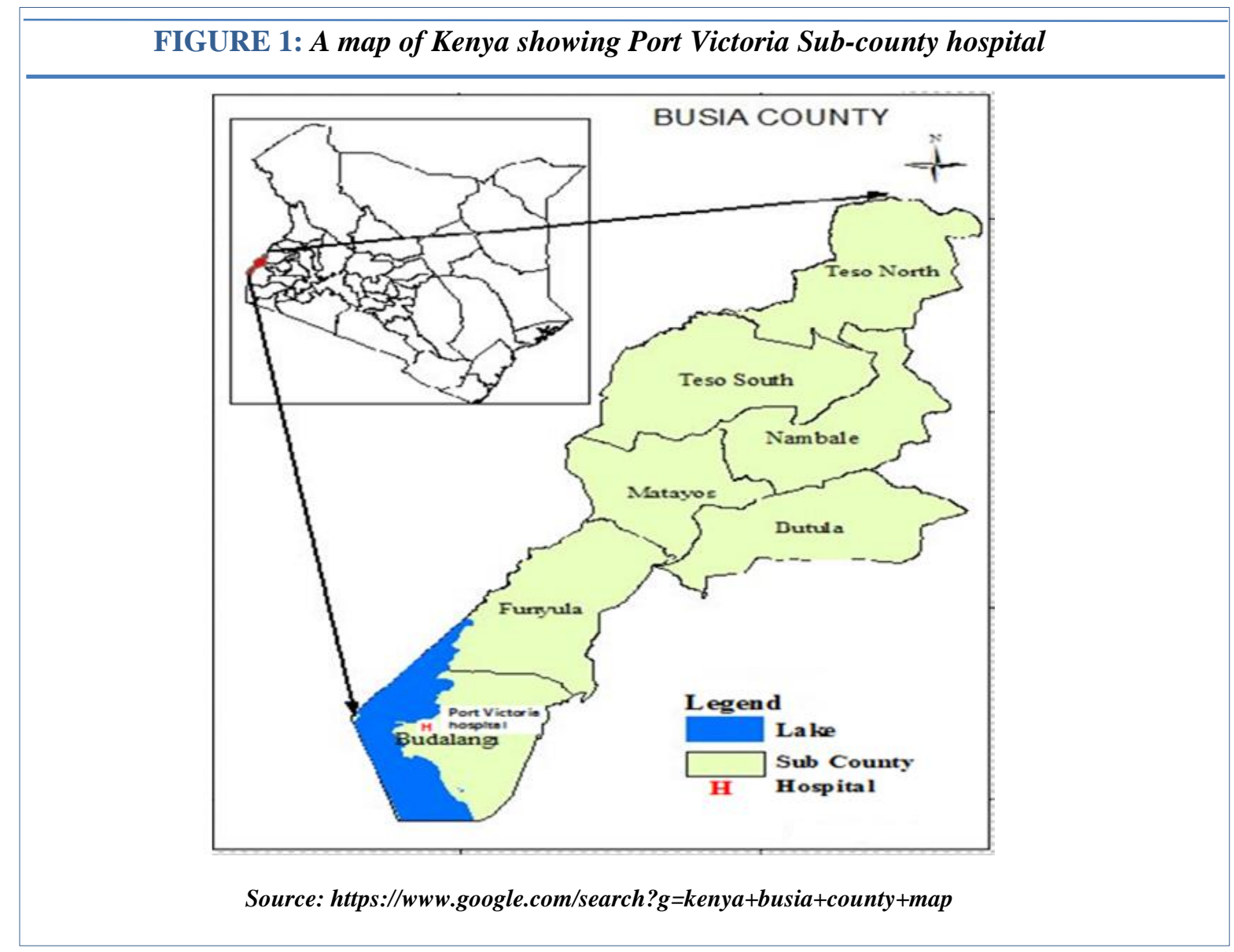

\section{Inclusion criteria}

Patients with diarrhea of three or more episodes per day, who are five years and above

Patients willing to consent to participate in the study.

\section{Exclusion Criteria}

Patients with diarrhea of less than three episodes per day and patients below five years of age Patients unwilling to consent to participate in the study.

\section{Sample determination}

The formula by fisher et al. 1998 was used to determine the minimum number of diarrheal patients to be sampled in this study. We suspected diarrheal illness is common perhaps $50 \%$ suspected cases during rainy season. The formula was

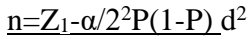

where;

$\mathrm{n}=$ Sample size $\mathrm{Z}_{1}-\alpha / 2^{2}=1.96 \mathrm{P}=$ Expected proportion in population $(50 \%)$

$\mathrm{d}=$ Absolute precision.

Diarrheal causing pathogen isolation rate was assumed to be $50 \%$ and level of significance was $5 \%$. Therefore, $\mathrm{P}=0.5$, $\mathrm{d}=0.05$ ( $5 \%$ absolute precision).
Therefore, $\mathrm{n}=1.96^{2} \times 0.5 \times(1-0.5) / 0.05^{2}=384.16$ patients. This was rounded to 400 stool samples from the same number of patients.

\section{Procedure for Study Participants}

All patients attending Port Victoria Sub-County Hospital and who met the inclusion criteria were sampled during rainy seasons (April to June) and August to September. A total of 100 samples were collected every year within these five months, 20 samples per months and an average of 1 sample per-day. The study purpose was explained to the patients. Those who accepted to participate in the study were requested to sign consent for recruitment.

A questionnaire was used to collect socio-demographic information such as age, gender, area of residence and occupation. Guardians/parents of minors/children were interviewed on their behalf. Patients were recruited from the outpatient clinic. We defined any diarrhea as the passage of three or more liquid stools within 24 hours, or any number (more than three times) of loose stools within 24 hours as per 
WHO definition. Stool samples were collected on the same day the patient arrived at the hospital with episodes of diarrhea. Identification of bacterial pathogens and antimicrobial susceptibility was conducted following standard microbiological processes.

\section{Specimen Collection}

Stool / rectal swabs were collected from patients presenting to the hospital with diarrhea during rainy season using sterile spatula and placed in sterile polypots. These were transferred into Carry Blair transport medium (Thero Fisher Scienific Inc, USA). The specimen tubes were transported in cooler boxes from the health facility to KEMRI, CIPDCR- Busia laboratories within 48 hours. After stool processing, the culture plates were decontaminated before disposal. The study subjects benefited from free diagnosis.

\section{Stool Processing}

\section{Microscopy Procedures}

Microscopy is a technique that involves the evaluation of a stool sample under Columbus microscope (Olympus BH-2, Optical Co. ltd, Japan) to determine the presence of ova and parasite that infect the lower digestive tract, causing symptoms such as diarrhea. Most parasites have more than one form through which they develop. Many have a mature form and a cyst and/or egg (ova) form. A small amount of the stool specimen was placed onto a microscope slide. Two drops of saline were added and mixed well and placed under a microscope. This was examined at $\mathrm{X} 40$ for presence or absence of ova or cyst.

\section{Microbiological Procedures}

A loopful of stool sample was inoculated into alkaline peptone water; $\mathrm{pH} 8.2$ and incubated for 8 hours at $37^{\circ} \mathrm{C}$. Subculture was done on TCBS (Central drug house ltd, India), XLD (Hardy diagnostics, USA) and Mackonkey (Oxoid, UK) and further incubated at $37^{\circ} \mathrm{C}$ for 24 hours. Direct culture was carried out on TCBS, XLD, Campylobacter blood free agar (Oxoid, UK), and enrichment in Selenite F medium (Himedia laboratories PVT ltd India) to isolate the bacterial pathogens. Rectal swabs were processed in the same manner.

\section{Identification of Bacterial Pathogens}

Standard methods for the identification of both Enterobacteriacae and non-entrobacteriacae was followed (Edward and Ewing). These included colony morphology on TCBS, XLD and Mackonkey; gram stain reaction and other biochemical tests like motility in saline, oxidase production and susceptibility to vibriostatic compound $\mathrm{O} / 129$ at 10 and 150 micrograms (Oxoid, USA). Further identification was done on API20NE and API20E. (API bioMerieux, Sa, France).

\section{Isolation and Characterization of Isolates}

Within a maximum of 2 hours upon arrival at the KEMRI laboratories, the stool samples/rectal swabs were plated on TCBS using cotton swab and left to incubate overnight at $37^{\circ} \mathrm{C}$. In the second day, TCBS plates were examined for growth of typical yellow or blue-green colonies. Further incubation was done for an additional 24 hours if no growth was identified.

Sensitivity to vibrio-static agent 0129

Typical colonies were inoculated on blood agar with a sterile wire loop. Filter discs containing the vibrio-static agent $\mathrm{O} 129,10 \mu \mathrm{g}$ and $150 \mu \mathrm{g}$, respectively were added to the agar surface. The resultant was incubated at room temperature for 30 minutes and then overnight at $37^{\circ} \mathrm{C}$.

\section{Oxidase test}

On day 3, an oxidase strip was placed in a clean Petri dish. Using a sterile applicator stick, colonies from the TCBS agar plate were taken and rubbed in the strip. The immediate development of deep blue or purple colour was a positive test result. If there was no colour change or weak colour change after 10 seconds, this was considered a negative test result. V. cholerae O1 NCTC 11218 as positive control and Escherichia coli NCTC 10418 as negative control were used.

\section{Motility Test Procedure}

Motility refers to ability of bacteria to change direction. This is important when bacteria require moving away or towards repellents or attractants respectively. Motile bacteria are effective root colonizers and can swim towards root exudates or other nutrient gradients earlier than nonmotile bacteria. A colony of a young (18 to 24 hour) culture growing on agar medium was touched with a sterile needle. This was stabbed down the center of the tube to about half the depth of the medium. This was then incubated at $35^{\circ} \mathrm{C}$ $37^{\circ} \mathrm{C}$ and examined daily for up to 7 days. Positive motility test was determined if organisms spread out into the medium from the site of inoculation.

A negative motility test was determined if the organisms remained at the site of inoculation. These Organisms were again placed on a microscope glass slide with a drop of distilled water; a similar slide was set as a control by adding a drop of saline. A small loop of culture was emulsified in saline using separate wires and this was covered by a glass cover slip. This was examined under $\mathrm{x} 40$ and oil immersion 
lenses and the presence/absence of motility were confirmed.

\section{Agglutination Test Procedure}

Agglutination is the clumping of cells such as bacteria or red blood cells in the presence of an antibody or complement. An agglutination test is carried out on slides and is called slide agglutination tests. Serotyping of bacteria is a good example of slide agglutination tests. For bacterial serotyping, a saline emulsion of the bacterial colony is made on a glass slide and a drop of specific antiserum is added and mixed. Antibodies bind to the antigens on the surface of bacteria and aggregate them resulting in visible clumps. One drop of saline and one drop of anti-sera was placed on microscope glass slide. The test organism was then emulsified (flame between each drop). The slide was rotated for 30 seconds and the presence of visible agglutination recorded. The presence of agglutination in the anti-sera denoted a positive reaction. Non- agglutination was observed in the saline portion.

\section{Drug Susceptibility Testing Procedure}

A susceptibility analysis is a test that determines the "sensitivity" of bacteria to an antibiotic. It also helps the doctor determine which drugs are likely to be most effective in treating the infection. A sterile wire loop was used to inoculate pure colonies of bacterial isolates onto Muller Hinton agar (Himedia Laboratories PVT Ltd, India) plate. About three to four different antibiotic disks was spread on each agar plate containing bacterial inoculums. The plate was sealed using a parafilm and incubated for 12-24 hours at $37^{\circ} \mathrm{C}$. Antibiotic inhibition zones were taken using a ruler.

The results noted down was interpreted either as sensitive or resistant depending on the standard measurement of a specific antibiotic disks.
Five antibiotics were used for susceptibility testing, Chloramphenicol, Ciprofloxacin, Ampicillin, Nalidixic acid and tetracycline.

\section{Data Analysis}

Collected data was cleaned and entered into a Microsoft excel for Windows database developed for this purpose. Analysis was done by SPSS version 20. Univariate analysis was carried out to summarize socio-demographic factors and other frequencies. Descriptive statistics was done to determine the frequency of bacterial pathogens from stool samples.

\section{Ethical considerations}

Ethical approval for this study (KEMRI/SSC 1503) was sought from the national ethical review committee at KEMRI. The study set-up and purpose were explained to the study participants/caregivers after which written informed consent was sought. For confidentiality purposes, patients' names were not used on labels, on forms, or elsewhere. Instead unique patient identification numbers were used for identification of samples.

\section{RESULTS}

Out of 400 participants (male, 187 and female, 213) had the median age of 20 years with a range of 5-60 years. A total of $171(42.8 \%)$ patients had no formal education, 123 $(30.8 \%)$ had attained primary education, $86(21.8 \%)$ had secondary education and $20(5.0 \%)$ had gone to college and university (tertiary education). $200(50 \%)$ patients were involved in fishing, $50(12.5 \% \%)$ were small scale traders, $20(5 \%)$ were small scale farmers and $30(7.5 \%)$ were fishing at the same time students and $50(12.5 \%)$ students and 50 (12.5\%) pupils (Table 1). The study sought to determine the spectrum of bacterial pathogen causing diarrhea among patients visiting Port Victoria hospital facility along Lake Victoria. From the findings, Non O1 Vibrio Cholerae and Salmonella pathogens seem to cause majority of diarrhea than the rest (Table 2). 
TABLE 1. Socio-demographic Information of Participants

\begin{tabular}{lccc}
\hline Age (years) & \multicolumn{2}{c}{ Gender } & Total (\%) \\
\hline & Male & Female & \\
$5-12$ & $78(19.5)$ & $72(18.0)$ & $150(37.5)$ \\
$13-17$ & $54(13.5)$ & $60(15.0)$ & $114(28.5)$ \\
$\geq 18$ & $55(13.8)$ & $81(20.3)$ & $136(34.0)$ \\
Total & $\mathbf{1 8 7}$ & $\mathbf{2 1 3}$ & $\mathbf{4 0 0}(\mathbf{1 0 0 . 0})$ \\
\hline \multicolumn{4}{c}{ Education } \\
\hline No Education & $71(17.8)$ & $100(25.0)$ & $171(42.8)$ \\
Primary & $59(14.8)$ & $64(16.0)$ & $123(30.8)$ \\
Secondary & $50(12.5)$ & $36(9.0)$ & $86(21.5)$ \\
Tertiary & $7(1.8)$ & $13(3.2)$ & $20(5.0)$ \\
Total & $\mathbf{1 8 7}$ & $\mathbf{2 1 3}$ & $\mathbf{4 0 0}$ \\
\hline \multicolumn{4}{c}{$\mathbf{O c c u p a t i o n}$} \\
\hline Fishing & $111(27.7)$ & $89(22.3)$ & $200(50.0)$ \\
Small scale traders & $35(8.7)$ & $15(3.8)$ & $50(12.5)$ \\
Small scale farmers & $6(1.5)$ & $14(3.5)$ & $20(5.0)$ \\
Fishing and students & $21(5.3)$ & $9(2.2)$ & $30(7.5)$ \\
Students & $22(5.5)$ & $28(7.0)$ & $50(12.5)$ \\
Pupils & $27(6.8)$ & $23(5.7)$ & $50(12.5)$ \\
\hline Total & $\mathbf{2 2 2}$ & $\mathbf{1 7 8}$ & $\mathbf{4 0 0}$ \\
\hline
\end{tabular}

TABLE 2. Number (\%) of Bacteria Pathogens Isolated and Parasites Identified $n=317$

\begin{tabular}{lc}
\hline Bacterial Pathogens & Frequencies n $(\%)$ \\
\hline Non O1 Vibrio Cholerae & $98(30.9)$ \\
Salmonella species & $97(30.6)$ \\
Shigella species & $47(14.8)$ \\
Aeromonas species & $38(12.0)$ \\
Aeromonas and Giardia lamblia & $3(0.9)$ \\
Giardia lamblia and Schistosoma mansoni & $11(3.5)$ \\
Giardia lambla & $6(1.9)$ \\
Others & $3(4.4)$ \\
\hline
\end{tabular}

Sensitivity patterns of bacterial species to commonly prescribed antibiotics were determined. out of 98 non $\mathrm{O} 1$ Vibrio cholerae isolates, $86.7 \%$ were sensitive to chloramphenicol; $76.5 \%$ to ciprofloxacin; $65.3 \%$ to Tetracycline; $63.3 \%$ to Ampicilin and $53.1 \%$ to Nalidixic acid. Sensitivity patterns of Salmonella species to commonly prescribed antibiotics were also determined. out of 97 Salmonella species isolates, $80.4 \%$ each were sensitive to chloramphenicol and ciprofloxacin; $79.4 \%$ to Tetracycline; $50.5 \%$ to Ampicilin and $70.1 \%$ to Nalidixic acid in. Out of 47 Shigella species isolates, $76.6 \%$ were sensitive to chloramphenicol; 74.5 to ciprofloxacin; $61.7 \%$ to Ampicilin; 70.7 to Nalidixic acid and $70.2 \%$ to Tetracycline. Sensitivity patterns of Aeromonas species to commonly prescribed antibiotics were determined. Majority of the bacteria responded well to Chloramphenicol, Ciprofloxacin and Nalidixic acid and poorly to ampicillin. Out of 41 Aeromonas species isolates, $73.2 \%$ were sensitive to chloramphenicol; $73.2 \%$ to ciprofloxacin; $53.7 \%$ to Ampicilin; 70.7 to Nalidixic acid and $68.3 \%$ to Tetracycline (Table 3). A total of 317 organisms (283 bacteria, 31 parasites and 3 others) were identified from 303 patients. These were: 98(30.9\%), Non O1 Vibrio cholera; 97(30.6\%), Salmonella species; 47(14.8.0\%), Shigella species; 38(12.0\%), Aeromonas species; 6(1.9\%), Giardia lambla. $3(0.9 \%)$, others. About $3(0.9 \%)$ had a mixed infection of 
Aeromonas species and Giardia lamblia and 11(3.5\%), Giardia lamblia and Schistosoma mansoni (Table 2). Sensitivity patterns of bacterial species to commonly prescribed antibiotics were determined. out of 98 non $\mathrm{O} 1$ Vibrio cholerae isolates, $86.7 \%$ were sensitive to chloramphenicol; $76.5 \%$ to ciprofloxacin; $65.3 \%$ to tetracycline; $63.3 \%$ to ampicilin and $53.1 \%$ to nalidixic acid. Sensitivity patterns of Salmonella species to antibiotics were as follows: out of 97 Salmonella species isolates, $80.4 \%$ each were sensitive to chloramphenicol and ciprofloxacin; $79.4 \%$ to Tetracycline; $50.5 \%$ to ampicilin and $70.1 \%$ to nalidixic acid. Out of 47 Shigella species isolates, $76.6 \%$ were sensitive to chloramphenicol; 74.5 to ciprofloxacin; $61.7 \%$ to ampicilin; 70.7 to nalidixic acid and $70.2 \%$ to Tetracycline. Sensitivity patterns of Aeromonas species to commonly prescribed antibiotics were determined. Majority of the bacteria responded well to chloramphenicol, ciprofloxacin and nalidixic acid and poorly to ampicillin. Out of 41 Aeromonas species isolates, $73.2 \%$ were sensitive to chloramphenicol; $73.2 \%$ to ciprofloxacin; $53.7 \%$ to ampicilin; 70.7 to nalidixic acid and $68.3 \%$ to tetracycline (Table 3).

TABLE 3. Susceptibility Pattern for Bacterial Isolates

\begin{tabular}{|c|c|}
\hline \multicolumn{2}{|c|}{ Non 01 Vibrio cholerae species $(\%) . \mathrm{n}=98$} \\
\hline Antibiotics & Sensitive \\
\hline Chloramphenicol & 85 (86.7) \\
\hline Ciprofloxacin & 75 (76.5) \\
\hline Ampicillin & $62(63.3)$ \\
\hline Nalidixic acid & $52(53.1)$ \\
\hline Tetracycline & $64(65.3)$ \\
\hline \multicolumn{2}{|c|}{ Salmonella species $(\%) . \mathrm{n}=97$} \\
\hline Chloramphenicol & $78(80.4)$ \\
\hline Ciprofloxacin & $78(80.4)$ \\
\hline Ampicillin & $49(50.5)$ \\
\hline Nalidixic acid & $68(70.1)$ \\
\hline Tetracycline & $77(79.4)$ \\
\hline \multicolumn{2}{|c|}{ Shigella species $(\%) . \mathrm{n}=47$} \\
\hline Chloramphenicol & $36(76.6)$ \\
\hline Ciprofloxacin & $35(74.5)$ \\
\hline Ampicillin & $29(61.7)$ \\
\hline Nalidixic acid & $39(83.0)$ \\
\hline Tetracycline & $33(70.2)$ \\
\hline \multicolumn{2}{|c|}{ Aeromonas species $(\%) . n=41$} \\
\hline Chloramphenicol & $30(73.2)$ \\
\hline Ciprofloxacin & $30(73.2)$ \\
\hline Ampicillin & $22(53.7)$ \\
\hline Nalidixic acid & $29(70.7)$ \\
\hline Tetracycline & $28(68.3)$ \\
\hline
\end{tabular}




\section{DISCUSSION}

The study was designed to determine pathogens isolated from patients with diarrhea who were five and more years living along Lake Victoria region of Kenya. In this study, the most affected were young individuals aged between 5 and 12 years. This is because this age bracket have poor personal hygiene including not washing hands before and after eating ${ }^{65}$. In this study, individuals with no or lower education were more likely to have diarrhea when compared with individuals with higher education. This finding was similar with other studies, where the prevalence of diarrhea varies according to education. Children whose mothers didn't know how to read and write ${ }^{66}$ had relatively high diarrhea cases than those who knew how to read and write. Thus education provides the knowledge on the rules of hygiene and clean feeding 67,68 .

Majority of the studied group regardless of the gender were fishermen and women. Exposure to drinking of contaminated untreated water directly from Lake Victoria ${ }^{45}$ during fishing might have contributed to transmission of known enteric pathogens which are passed through contaminated food and water. In this study, four types of bacterial pathogens were isolated and two parasitic pathogens were identified. These bacterial pathogens were Non O1 Vibrio cholerae, Salmonella species, Shigella species and Aeromonas species. As much as the diarrheal infection is a common problem worldwide $^{69}$, the isolation of the first three bacteria from patients with diarrhea have been reported in previous studies including Kenya ${ }^{24,43,60,70,71,72}$.

In this study Non O1 Vibrio cholera 98(30.9\%) were the leading isolates causing diarrhea in the studied population. The ecosystem of Non O1 Vibrio cholerae being Salty and brackish environment ${ }^{73}$ contradicts the existence of the bacteria in fresh waters of Lake Victoria. However, human activities may alter water ecosystems ${ }^{74}$. Lake Victoria is well characterized with extensive fishing ${ }^{75}$ and this is evident from this study that majority of diarrheal patients were fishermen and women. Pollution of the Lake Victoria with discharge of raw sewage dumping of domestic and industrial waste, fertilizer and chemicals from farms which in turn increase the invasive water hyacinth ${ }^{76}$ may alter the salinity of waters thus favoring the existence of these bacteria. Evaporation throughout the year due to high temperatures along Lake Victoria may lead to relatively increased salinity in freshwater bodies. In addition non $\mathrm{O} 1$ Vibrio cholera possess additional virulence factors that enable it to survive in various ecosystems ${ }^{77}$. Although, studies carried out in other places of Kenya have reported the isolation of serotypes of Vibrio cholera pathogen from stool of both children and adults $^{45,60,24,62}$.
The prevalence of these Vibrio species was lower especially among the children compared to the current study which constituted both children and adults. The prevalence difference could be due to groups of study participants where the previous obtained samples from children and the current from both the children and adults. Other studies in Kenya have reported isolation of this pathogen from environmental samples ${ }^{61},{ }^{62}$. Another study carried out in Nigeria reported lower isolation of non O1 Vibrio cholera from only adult patients with acute diarrhea compared to the current ${ }^{14}$ which studied all patients with three or more episodes of diarrhea per day excluding patients with two or one episodes of diarrhea per day.

Salmonella typhi causes typhoid fever which is spread by eating or drinking food or water contaminated with the feces of an infected person. Salmonella species is associated with poor sanitation and poor hygiene ${ }^{78}$. Port Victoria being among the areas in Kenya with poor sanitary condition may have contributed to existence of these bacteria. The finding of this investigation also confirms earlier observations of the isolation of Salmonella from patient with diarrhea elsewhere ${ }^{79,80,81}$. In this study, Salmonella species was the second prevalent organism. In comparison with other studies carried out elsewhere, the prevalence of this organism in other studies carried out in Kenya ${ }^{45,39,40,43,60}$, Korea ${ }^{18}$ and Ethiopia $^{81}$ were lower compared to the current study. This difference could exist because previous studies considered characterization of Salmonella species into specific serotypes compared to the current which did not characterize Salmonella species into various serotypes types.

Shigella species causes human dysentery in $\operatorname{man}^{82,83}$. Report from the World Health Organization is that, the bacteria leads in the cause of diarrhea in human ${ }^{84}$ worldwide. In this study, Shigella species was third affecting 47(14.8\%) patients presenting in the health facility with diarrhea. Shigella species has been isolated from stool of patients with diarrhea in Masaai communities in Kenya ${ }^{70}$. Consumption of meat by Masaai communities and fish by the participants in the current study might have served as associated factors for Shigella infection since this pathogen is known to be zoonotic in nature. Other previous studies have also reported Shigella species to cause travelers' diarrhea ${ }^{85,26,86}$, however the current study reports Shigella species to cause diarrhea in the domestic population who did not have a history of travel for the last one year. Isolation of Aeromonas species from clinical samples is of great concern. This bacterium was first reported from a river sample in Kenya ${ }^{48}$. However, in this study was least isolated from patients with diarrhea. In other previous studies carried out elsewhere, this bacterium was isolated from travelers with diarrhea, healthy carriers ${ }^{72}$ and immune-compromised individuals due to $\mathrm{HIV}^{87,88}$. 
However, in the current study the patient had no history of travel for the last one year and their HIV status was not determined. Its isolation in the current study is linked to the fact that majority of patients were fishermen and women who come into contact with and drink water directly from the lake during fishing. This might have served as a risk factor for its transmission as evidenced by the fact that, Aeromonas species had been isolated from a fresh water river samples in Kenya $^{48}$ and elsewhere ${ }^{89}$. In the current study, all isolated pathogens were subjected to five commonly prescribed antibiotics. Sensitivity pattern showed varied sensitivities to all tested antimicrobials. Hence, there was variation in drug sensitivity patterns among all isolates compared to other studies carried out elsewhere. As much as over $50 \%$ of all the isolates were sensitive to all tested antimicrobials. The resistance shown by these pathogens still poses a threat in their management. In the current study, the predominance of Non O1 Vibrio cholera from diarrheal patients probably reflects a high prevalence in the community.

Tetracycline and ciprofloxacin among others have been generally considered as the drug of choice for the treatment of cholera. However, the resistance profiles indicate that these antibiotics will be less effective for treating non-01 $\mathrm{V}$. cholera infections. In deed comparing with another previous study in Kenya ${ }^{61}$, the resistance of this pathogen against tetracycline in the current study is slightly higher than the previous ${ }^{61}$. However the resistance of ciprofloxacin against Vibrio species was lower in the current study than the previous because this drug is expensive for the population in the studied site and it is not purchased over the counter ${ }^{61}$. In another study conducted along Lake Victoria, Kenya more than 10 years ago showed that, all Vibrio species $^{45}$ isolated were susceptible to all antibiotics that were tested in this study. This difference in antibiotic susceptibility is that the previous study considered patients who had only bloody diarrhea while the current considered all the patients with diarrhea. To add on this, the previous study was carried out more than 10 years ago of which resistance might have developed along the way. In another study carried out elsewhere ${ }^{90}$, Vibrio species reported lower resistance to tetracycline and ciprofloxacin, perhaps because it was not the drug of choice to treat infection caused by this pathogen. In the current study, resistances of Vibrio Cholerae Non O1 was slightly more against Nalidicic acid and slightly lower against Ampicillin compared to the previous study, $30 \%$ and $42.5 \%$ respectively in $\mathrm{China}^{90}$. This difference in susceptibility could be due to geographical location of the current and previous studies as it is ascertained by other studies that resistances of bacteria are different basing of geographical distribution ${ }^{91}$.
The current study identified and determined susceptibilities of Salmonella species against various antibiotic. Generally, susceptibilities to chloramphenicol, ciprofloxacin and tetracycline were excellent, while susceptibility of Nalidixic acid and Ampicilin were fairly good. However, resistances of some isolates to these antibiotics in the current study are of great concern especially in the studied individuals, because these may rise with time. The current study noted some differences in all the five antibiotics tested with previous study carried out in Kenya ${ }^{37}$ where by all isolates of Salmonella species from sixteen adults were susceptible to ciprofloxacin, chloramphenicol and nalidixic acid while $82 \%$ of the isolates from 22 children were resistant to ampicillin, tetracycline and chloramphenicol. This difference could be explained by the fact that the previous study considered fewer blood culture samples compared to the sample size of stool cultures in the current study. There was also variation in antibiotic resistance and susceptibilities of all these tested antibiotics against Salmonella isolates between the current study and the previous study in Kenya ${ }^{38}$. The difference could be due to the fact that the previous study obtained its samples from an outbreak which probably had different species of Salmonella from the current. In another study carried out in Kenya ${ }^{41}$, all Salmonella isolates were sensitive to Ciprofloxacin while in the current study, some isolates were resistant to this drug. This is because previous study was carried out more than 10 years ago of which resistance might have developed along the way. The current study shows that $77(79.4 \%)$ and 49(50.5\%) Salmonella isolates are sensitive to Tetracycline and Ampicillin while the previous study shows all to be resistant ${ }^{92}$. The previous study obtained samples from poultry while the current from human. About 93.1\% of Salmonella species were resistant to both Tetracycline and Ampicillin in a previous study ${ }^{93}$. The previous study considered both samples from both human and animals while the current samples were from only human. On the other hand, study conducted in India reported that $78.4 \%$ S. typhi showed resistance to chloramphenicol which is conventionally used to treat typhoid fever ${ }^{94}$, the current study shows opposite results in that $80.4 \%$ are sensitive to chloramphenicol which is again frequently prescribed in the treatment of typhoid cases.

In this study, testing of Shigella species against five antibiotics has revealed various susceptibility and resistance patterns. Both patterns have revealed differences in the current and previous studies in Kenya. In the current study susceptibility of this pathogen is lower to ciprofloxacin and higher to ampicillin and tetracycline than the previous study $^{24}$. 
In the current study susceptibility of this pathogen is lower to ciprofloxacin and nalidixic acid and higher to chloramphenicol, ampicillin and tetracycline than the previous study ${ }^{45},{ }^{60}$. The differences between the current and the previous studies is that, the previous studies obtained their samples from children while the current considered both children and adults. As the antimicrobial sensitivity test results imply, $74.5 \%$ and $83.0 \%$ of the Shigella species isolates to be sensitive to two antimicrobials: ciprofloxacin and nalidixic acid respectively. Differently, results have been reported from Ethiopia where $100 \%$ Shigella species isolates were found sensitive $\mathrm{e}^{81}$ and $96.4 \%$ Shigella species isolates in China were resistant to nalidixic acid ${ }^{95}$. Sensitivity to ciprofloxacin in the present study is slightly lower than the result found from Gondar University where $91.1 \%$ were found to be sensitive ${ }^{96}$. In comparison to a previous study in Tehran, the percentage of resistance of Shigellla species against ampicillin is slightly lower with the current study which was $10 \%{ }^{97}$. In the present study only $10(21.3 \%$ ) of the isolates are resistant to tetracycline and this does not agree with related studies in Iran, India, Chile, and Nepal whose resistance was more than $50.0 \%{ }^{47,98,99,100,101}$. Study done in India found all Shigella species isolates sensitive to Chloramphenicol ${ }^{102}$, while in the current study, 36(76.6\%) were sensitive and $6(12.8 \%)$ resistant. The findings of the current study do not agree with the previous Uzbekistan where some strains of Shigella flexneri, 28(90.4\%) and Shigella sonnei $1(4.8 \%)$ were resistant ${ }^{103}$. The resistance differences between the current and the previous studies could be due to heritable factors that determines intestinal environment ${ }^{87}$ thus heritable factors of the studied individuals in the previous studies are different from the individuals in this study.

More than a half of the isolated Aerononas species were susceptible to all antibiotics except Ampicillin whose susceptibility was almost half. Unlike in the previous study whose all the isolates were susceptible to Ciprofloxacin ${ }^{89}$, only $30(73.2 \%)$ in the current study were susceptible. This difference could be attributed by the fact that the previous study considered specimen from environment and clinical sources while the current concentrated on the clinical samples only. Susceptibility of Aeromonas species to Tetracycline in the current study was lower than in the previous study $94.4 \%{ }^{89}$. The data from the present study indicated $53.7 \%$ of Aeromonas species being susceptible to Ampicillin a situation that does not concur with results from sensitivity pattern of the previous study that showed varied sensitivities to all tested antimicrobials ${ }^{104}$. The previous study had $88 \%$ some biotype resisting ampicillin and $100 \%$ other biotype susceptible. The reason for varied susceptibilities in the previous study was that resistant biotype are frequently isolated than the susceptible ones a situation that could be related to our case. As much as in the current study indicate Aeromonas species as the least isolated pathogen from patients with diarrhea still some $17.1 \%$ and $14.6 \%$ were resistant to chloramphenicol and Nalidixic acid respectively the reason of which could be these antibiotics are still commonly prescribed in typhoid cases.

\section{Limitation of the study}

This study did not consider HIV status of the participants because the study was interested to know the type of organisms that cause diarrhea regardless of whether patients had HIV or not. It did not also analyze antibiotic testing in relation to sex and age rather it generalized the analysis. The study also did not determine whether diarrhea was caused by the virus to check the 97 samples without parasite or bacteria whether they had viruses or not.

\section{CONCLUSION}

Bacterial isolates in this study responded differently to antibiotics as compared to other previously done studies. The susceptibilities expressed were fairly good; however, some of the resistances shown pose a threat to public health, especially in unexposed populations. With the observed trends, bacterial species in different geographical settings have responded differently to antibiotics. It may be beneficial for clinicians to consider antibiotic susceptibility testing prior to management of patients to slow down resistance development.

Acknowledgement: We thank the study participants and all staff members of Port Victoria hospital who assisted in data collection. This work was supported by financial assistance from KEMRI- Internal Research Grant. These findings are published with the approval of the Director, Kenya Medical Research Institute

\section{REFERENCES}

1. WHO. The global burden of diseases. Updates. In: Geneva Switzerland; 2004.

2. Lozano R, Naghavi M, Foreman K, et al. Global and regional mortality from 235 causes of death for 20 age groups in 1990 and 2010: A systematic analysis for the Global Burden of Disease Study 2010. Lancet. 2012;380(9859):2095-2128. doi:10.1016/S0140-6736(12)61728-0

3. UNICEF/WHO. Global, regional, and national causes of child mortality: an updated systematic analysis for 2010 with time trends since 2000. Lancet. 2012;380(9850):2095-2128.

4. David Hemson, Fritz Scheuren. WHO: Water and hygiene interventions Acad Journals. 2005;6(27).

5. Jamison Dean T, Feachem Richard G, Makgoba Malegapuru W, et al. Disease and Mortality in Sub-Saharan Africa. In: Diarrheal Diseases. Chapter 9. 2nd Edition. ; 2005:139-178.

6. Robert C. Reiner, Nicholas Graetz, Daniel C. Casey, Christopher Troeger, Gregory M. Garcia, Jonathan F. Mosser., Aniruddha Deshpande, Scott J. Swartz, Sarah E. Ray, Brigette F. Blacker, Puja C. Rao, Aaron OsgoodZimmerman, Roy Burstein DMP, Ian M. Davis, Ian D. Letourneau, Lucas 
Earl, Jennifer M. Ross, Ibrahim A. Khalil, Tamer H. Farag, Oliver J. Brady, Moritz U.G. Kraemer, David L. Smith, Samir Bhatt, Daniel J. Weiss, Peter W. Gething, Nicholas J. Kassebaum, Ali H. Mokdad, Christopher J.L. N. Variation in Childhood Diarrheal Morbidity and Mortality in Africa, 2000-2015. Engl J Med. 2018;379:1128-1138.

7. Elmi, O.E., and Dioso PR. Prevalence of Diarrhoeal Diseases Among Children under Five Years in East African Countries from 2012-2017. ASEAN J Sci Technol Dev. 2017;34(1):51-55.

8. Jeffrey A.Tornheim, Ayub S.Manya, NorbertOyando, et al. The epidemiology of hospitalization with diarrhea in rural Kenya: the utility of existing health facility data in developing countries. Int J Infect Dis. 2010;14(6):e499-e505

9. Mugoya I, Kariuki S, Galgalo T, et al. The rapid spread of Vibrio cholerae O1 throughout Kenya, 2005. Am J Trop Med Hyg. 2008;78:527-533.

10. Ahmed Abade Mohamed, Joseph Oundo, Samuel M. Kariuki, et al. Molecular Epidemiology of Geographically Dispersed Vibrio cholerae, Kenya, January 2009-May 2010. Emerg Infect Dis. 2012;18(6):925-931.

11. O-Tipo Shikanga, David Mutonga, Mohammed Abade, et al. High Mortality in a Cholera Outbreak in Western Kenya after Post-Election Violence in 2008. Am Soc Trop Med Hyg. 2009;81:1085-1090.

12. Jill W AHS, Wenjing Tao, Jenny Lofgren, Birgerc F. Diarrhoeal diseases in low and middle income countries, incidence, prevention and management. Infect Dis J. 2010;4 ()(133):113-124.

13. Al-Gallas N., Bahri O., Bouratbeen A., Ben Haasen A., Ben Aissa R. Etiology of acute diarrhoea in children and adults in Tunis, Tunisia, with emphasis on diarrhoeagenic Escherichia coli: prevalence, phenotyping, and molecular epidemiology. Am J Trop Med Hyg. 2007;77:571-582.

14. Okeke, I. N., O. Ojo, A. Lamikanra, Kaper J. Etiology of acute diarrhea in adults in southwestern Nigeria. J Clin Microbiol. 2003;41:4525-4530.

15. Salazar De Vegas, E. Z., B. Nieves, M. Ruiz, J. Ruiz, J. Vila MA, Velazco E. Molecular epidemiology and characterization of resistance mechanisms to various antimicrobial agents in Acinetobacter baumannii isolated in Mérida, Venezuela. Med Sci Monit. 2007;13:BR89-BR94.

16. Hyams KC, MK, Wolf, DN Taylor, Boedeker E, et al. Characterization of enterotoxigenic Escherichia coli isolated from US troops deployed to the Middle East. J Clin Microbiol. 1993;31(4):851-856.

17. Selinger CP, Greer S, Sutton C. Is gastrointestinal endoscopy a risk factor for Clostridium difficile associated diarrhea? Am J Infect Control. 2010;38(7):581-582

18. Kim NO, Jung SM, Na HY, et al. Enteric Bacteria Isolated from Diarrheal Patients in Korea in 2014. Osong Public Heal Res Perspect. 2015;6(4):233-240.

19. Tam CC, Rodrigues LC, Viviani L, et al. Longitudinal study of infectious intestinal disease in the UK (IID2 study): incidence in the community and presenting to general practice. Gut. 61(1):69-77.

20. Bryce J, Boschi-Pinto C, Shibuya K, Black RE. WHO Child Health Epidemiology Reference Group estimates of the causes of death in children. Lancet 365. 2005;365:1147-1152

21.Botelho B. A., Bando S. Y., Trabulsi L. R., Moreira-Filho C. A. Identification of EPEC and non-EPEC serotypes in the EPEC O serogroups by PCR -RFLP analysis of the fliCgene. J Microbiol. 2003;54:87-93.

22.Keskimaki M., Marjut E., Heidi P., Tarja H. EPEC, EAEA and STEC in stool specimens: Prevalence and molecular epidemiology of isolates. Diag Microbiol Infect Dis. 2001;40:151-15.

23. Rao M. R., Abu -Elyazeed R., Savarino S. J., et al. High disease burden of diarrhoea due to enterotoxigenic Escherichia coli among rural Egyptian infants and young children. J Clin Microbiol. 2003;41:4862-4864.

24. John T. Brooks, John Benjamin Ochieng, Kumar Lata, et al. Surveillance for Bacterial Diarrhea and Antimicrobial Resistance in Rural Western Kenya, 1997-2003. Clin Infect Dis. 2006;43:393-401.

25. Vilchez S, Daniel R, Margarita P, Filemon B, Roland M, Andrej W. Prevalence of diarrhoeagenic Escherichia coli in children from León, Nicaragua. J Med Microbiol. 2009;58:630-637.

26. Castelli F, Pezzoli C, L., Tomasoni. Epidemiology of travelers' diarrhea. J Travel Med. 2001;8(suppl 2):S26-S30.

27. Khalil IA, Troeger C, Blacker BF, et al. Morbidity and mortality due to shigella and enterotoxigenic Escherichia coli diarrhoea: the Global Burden of Disease Study 1990-2016. Lancet Infect Dis. 2018;18(11):1229-1240. doi:10.1016/S1473-3099(18)30475-4

28. Sánchez-Capilla AD, Sorlózano-Puerto A, Rodríguez-Granger J, Martínez-Brocal A, Navarro-Marí JM, Gutiérrez-Fernández J. Infectious etiology of diarrheas studied in a third-level hospital during a five-year period. Rev Esp Enfermedades Dig. 2015;107(2):89-97.

29. Vasco G, Trueba G, Atherton R, et al. Identifying etiological agents causing diarrhea in low income Ecuadorian communities. Am J Trop Med Hyg. 2014;91(3):563-569. doi:10.4269/ajtmh.13-0744

30. Bicer S, Col D, Erdag GC, et al. A retrospective analysis of acute gastroenteritis agents in children admitted to a university hospital pediatric emergency unit. Jundishapur J Microbiol. 2014;7(4):1-7. doi:10.5812/jjm.9148

31. Kotloff KL. The Burden and Etiology of Diarrheal Illness in Developing Countries. Pediatr Clin N Am. 2017;64:799-814.

32. Farah Naz Qamar, Muhammad Imran Nisar, Farheen Quadri, et al. Aeromonas-Associated Diarrhea in Children under 5 Years: The GEMS Experience. Am J Trop Med Hyg. 2016;95(4):774-780.

33. Gascón J, Vargas M, Schellenberg D, et al. Diarrhea in children under 5 years of age from Ifakara, tanzania: A case-control study. J Clin Microbiol. 2000;38(12):4459-4462. doi:10.1128/jcm.38.12.4459-4462.2000

34. Kariuki S., G. Revathi, N. Kariuki, et al. Invasive multidrug-resistant non-typhoidal Salmonella infections in Africa: zoonotic or anthroponotic transmission? J Med Microbiol. 2006;55:585-591.

35. Soto, S. M., M. A. Gonzalez-Hevia, Mendoza M. C. Antimicrobial resistance in clinical isolates of Salmonella enterica serotype Enteritidis: relationships between mutations conferring quinolone resistance, integrons, plasmids and genetic types. J Antimicrob Chemother. 2003;51:1287-1291.

36. Velge P., A. Cloeckaert, Barrow P. Emergence of Salmonella epidemics: the problems related to Salmonella enterica serotype Enteritidis and multiple antibiotic resistance in other major serotypes. Vet Res. 2005;36:267-288.

37. Kariuki S, Gilks C, Revathi G, C Hart. Genotypic analysis of multidrugresistant Salmonella enterica Serovartyphi, Kenya. Emerg Infect Dis. 2000;6:649-51.

38. Kariuki S, G, Revathi, Muyodi J, Mwituria J, Munyalo A, et al. Characterization of multidrug-resistant typhoid outbreaks in Kenya. $J$ ClinMicrobiol. 2004;42(4):1477-1482. doi:10.1128/JCM

39. Kariuki S, G, Revathi, Kariuki N, et al. Increasing prevalence of multidrug-resistant non-typhoidal salmonellae, Kenya, 1994-2003. Int J Antimicrob Agents. 2005;25:38-43.

40. Kariuki S, G, Revathi, Kiiru J, Lowe B, Berkley J, C Hart. Decreasing prevalence of antimicrobial resistance in non-typhoidal Salmonella isolated from children with bacteraemia in a rural district hospital, Kenya. Int $J$ Antimicrob Agents. 2006;28:166-171.

41. Onyango D, F, Machoni, Kakai R, EN Waindi. Multidrug resistance of Salmonella entericaserovarsTyphi and Typhimurium isolated from clinical samples at two rural hospitals in Western Kenya. J Infect Dev Ctries. 2008;2:106-111

42. Kariuki S, Oundo J, Muyodi J, B L, E, Threlfall, C Hart. Genotypes of multidrug resistant Salmonella enterica serotype Typhimurium from two regions of Kenya. FEMS Immunol Med Microbiol. 2000;Med Microb(29):9-13.

43. Brent AJ, JO Oundo, Mwangi I, L, Ochola, Lowe B, JA Berkley. Salmonella bacteremia in Kenyan children. Pediatr. Pediatr Infect Dis J. 2006;25(3):230-236

44. Roger L. Shapiro, Lata Kumar, Penny Phillips-Howard, et al. Antimicrobial-Resistant Bacterial Diarrhea in Rural Western Kenya. $J$ Infect Dis. 2001;183(11):1701-1704.

45. John B., Rogger L., Lata K., Joy G., Wells P. Epidemiology of sporadic bloody diarrhoea in Rural Western Kenya. Amer SocCF Trop Med Hyg. 2003;86:671-677.

46. Henry N. Njuguna, Cosmas Leonard, John Williamson, et al. Use of Population-based Surveillance to Define the High Incidence of Shigellosis in an Urban Slum in Nairobi, Kenya. PLoS One. 2013;8(3):e58437.

47. Juma BW, Kariuki S, Bulimo WD, WM M, Wurapa EK, PG, Waiyaki. Antibiotic susceptibility profiles of bacteria isolates obtained from patients 
presenting with diarrhoea in Machakos Distric Hospital, Kenya. East Cent Africa Med J. 2015;2:60-63.

48. Njeru S. Ngoci, Kiruki Silas, Limo Moses, et al. Antimicrobial resistance and plasmid profiles of Aeromonas hydrophilaisolated from River Njoro,Kenya. African J Biotechnol. 2012;11(96):16284-16290.

49. Sinha S, T. Shimada, Ramamurthy T, et al. Prevalence, serotype distribution, antibioticsusceptibility and genetic profiles of mesophilicAeromonasspecies isolated from hospitalizeddiarrhoeal cases in Kolkata, India. J Med Microbiol. 2004;53:527-534.

50. Lima A.A. Tropical diarrhea: New developments in traveler's diarrhea Curr Opin Infect Dis. 2001;14(5):547-552.

51. Ansari M, Raissy M. In vitro susceptibility of commonly used antibiotics against Vibrio spp. isolated from Lobster (Panulirushomarus). African J Microbiol Res. 2010;4(23):2629-2631.

52. Moira A. Gilliver, Bennett Malcolm, Michael Begon, Sarah M Hazel, Anthony Hart C. Enterobacteria: Antibiotic resistance found in wild rodents. Nature. 1999;401:233-234.

53. Paterson DL. Resistance in gram-negative bacteria: enterobacteriaceae. Am J Med. 2006;119(6 Suppl 1)):S20-8; discussion S62-70.

54. Ritchie DJ, Alexander BT, Finnegan PM. New antimicrobial agents for use in the intensive care unit. Infect Dis Clin North Am. 2009;23(3):665681.

55. Bhattacharya SK. An Evaluation of current cholera treatment. Expert OpinPharmacother. 2003;4:141-146.

56. Chiang SR, Chuang YC. Vibrio vulnificus infection: Clinical manifestation, pathogenesis and antimicrobial therapy. J Microbiol Infect. 2003;36:81-88.

57. Ahmed AM., Nakagawa, T., Arakawa, E., Ramamurthy T, Shinoda S., Shimamoto T. New aminoglycoside acetyltransferase gene, aac(3)-Id, in a class 1 integron from a multiresistant strain of Vibrio fluvialis isolated from an infant aged 6 months. J Antimicrob Chemother. 2004;53(6):947951.

58. Ceccarelli D., Salvia M., Sami J., Cappuccinelli P., Maria M. Colombo New Cluster of Plasmid-Located Class 1 Integrons in Vibrio cholerae O1 and a dfrA 15 Cassette -Containing Integron in Vibrio parahaemolyticus Isolated in Angola. Antimicrob Agents Chemother. 2006;50(7):2493-2499.

59. Okoh, A. I., Igbinosa, E. O. Antibiotic susceptibility profiles of some Vibrio strains isolated from wastewater final effluents in a rural community of the Eastern Cape Province of South Africa. BMC Microbiol. 2010;10(143):1-6

60. Sang WK, Oundo V, Schnabel D. Prevalence and antibiotic resistance of bacterial pathogens isolated from childhood diarrhoea in four provinces of Kenya. J Infect Dev Ctries. 2012;23(7):572-578.

61. Nyamboya Rosemary Atieno, Okemo Paul Owuor, Ombori Omwoyo. Isolation of High Antibiotic Resistant Fecal Bacteria Indicators, Salmonella and Vibrio Species from Raw Abattoirs Sewage in Peri-Urban Locations of Nairobi, Kenya. Greener J Biol Sci. 2013;3(5):172-178.

62. Kiiru J, Mutreja A, Mohamed AA, et al. A Study on the Geophylogeny of Clinical and Environmental Vibrio cholerae in Kenya. PLoS One. 2013;8(9):e74829.

63. Phavichitr N., A. Catto-Smith. Acute gastroenteritis in children: what role for antibacterials? Drugs. 2003;5:279-290.

64. Christopher PR, David KV, John SM, Sankarapandian V. Antibiotic therapy for Shigella dysentery. Cochrane Database Syst Rev. 2010;4:CD006784

65. ALBashtawy M. Personal hygiene in school children aged 6-12 years in Jordan. Br J Sch Nurs. 2015;10(8):395-398.

66. Anteneh A, Kumie A. Assessment of the impact of latrine utilization on diarrheal diseases in the rural community of Hulet Ejju Enessie Woreda, East Gojjam Zone, Amhara Region. Ethiop J Heal Dev. 2010;24(2):110118.

67. Shikur M, T, Marelign, T Dessalegn. Morbidity and associated factors of diarrheal diseases among under five children in Arba-Minch district, Southern Ethiopia. Sci J Public Heal. 2013;1(2):102-106.

68. Yilgwan C, G, Yilgwan, I., Abok. Domestic water sourcing and the risk of diarrhea: a cross-sectional survey of a semi-urban community in Nigeria. J Med. 2005;5(1):34-37.

69. Rafael Lozano MN, K, Foreman, Lim S, K Shibuya. Global and regional mortality from 235 causes of death for 20 age groups in 1990 and 2010: a systematic analysis for the Global Burden of Disease Study 2010. Lancet. 2012;380:2095-2128

70. Sang W, Kariuki S, Schnabel D, Boga H, Wamae C. Antibiotic susceptibility of Enteric pathogens from the Maasai community, Narok and Kajiado Districts, Kenya. African J Heal Sci. 2011;19:74-79.

71. Devarati Dutta, Goutam Chowdhury, Gururaja P. Pazhani, et al. Vibrio cholerae Non-O1, Non-O139 Serogroups and Cholera-like Diarrhea, Kolkata. India Emerg Infect Dis. 2013;19(3):464-467.

72. Jordi Vila, Ruiz, Joaquin, Francisco Gallardo, et al. Aeromonas spp. and Traveler's Diarrhea: Clinical Features and Antimicrobial Resistance. Emerg Infect Dis. 2003;9(5):552-554.

73. Jane-Francis Tatah Kihla Akoachere, Mbuntcha, Christelle Kwedjeu Pulcherie. Water sources as reservoirs of Vibrio choleraeO1 and non-O1 strains in Bepanda, Douala (Cameroon): relationship between isolation and physico-chemical factors. BMC Infect Dis BMC Ser \& open, Incl Trust. 2014;14:421.

74. Baquero F, Martínez J-L, Cantón R. Antibiotics and antibiotic resistance in water environments. Curr Opin Biotechnol. 2008;19:260-265.

75. Lake Victoria Fisheries Organisation. Regional report on Lake Victoria Frame surveys for 2000, 2002 and 2004. Jinja, Uganda. LVFO Lake Victoria Environ Manag Proj. 2005

76. National Aeronautics and Space Administration. "Water Hyacinth Reinvades Lake Victoria." Image Dat (NASA). 2007.

77. Chatterjee S, Ghosh K, Raychoudhuri A, Chowdhury G, Bhattacharya MK, et al. Incidence, virulence factors, and clonality among clinical strains of non-O1, non-O139 Vibrio cholerae isolates from hospitalized diarrheal patients in Kolkata. India J Clin Microbiol. 2009;47:1087-1095.

78. Wain J, Hendriksen RS, Mikoleit ML, Keddy KH, Ochiai RL. Typhoid fever. Lancet. 2015;385(9973):2236-2245.

79. Awole M, Gebre-Selassie S, Kassa T, Kibru G. Isolation of potential bacterial pathogens from the stool of HIV-infected and HIV-non-infected patients and their antmicrobial susceptibility patterns in Jimma Hospital, south west Ethiopia. Ethiop Med J. 2002;40(4):353-364.

80. Bo Svenungsson, Lagergren Åsa, Erik Ekwall, et al. Enteropathogens in Adult Patients with Diarrhea and Healthy Control Subjects: A 1-Year Prospective Study in a Swedish Clinic for Infectious Diseases. Clin Infect Dis. 2016;30(5):770-777.

81. Tadesse Eguale, Wondwossen A. Gebreyes, Daniel Asrat, Haile Alemayehu, Gunn, John S, Ephrem Engidawork. Non-typhoidalSalmonella serotypes, antimicrobial resistance and co-infection with parasites among patients with diarrhea and other gastrointestinal complaints in Addis Ababa, Ethiopia. BMC Infect Dis. 2015;15:497.

82. Pond K. "Shigella".Water recreation and disease. Plausibility of associated infections: Acute effects, sequelae and mortality. WHO $p p$ 2005:113-8. ISBN 978-92-4-156305-5.

83. Mims Cedric, Hazel Dockrell, Richard Goering, Roitt Ivan, Wakelin Derek, Zuckerman Mark. Medical Microbiology (3rd Ed.).; 2004

84. World Health Organization. Shigellosis".State of the art of new vaccine research and development. Immunization,. Vaccines Biol. 2006:10-12.

85. Sonnenburg von F, Tornieporth N, Waiyaki P, et al. Risk and aetiology of diarrhoea at various tourist destinations. Lancet. 2000;356:133-134.

86. Johnnie Yates, CIWEC Clinic Travel Medicine Center Kathmandu Nepal. Traveler's Diarrhea. Am Fam Physician. 2005;71(11):2095-2100.

87. Daskalov H. "The importance of Aeromonas hydrophila in food safety,." Food Control. 2006;176(6):474-483.

88. Whitaker Iain S, Kamya Cyril, A, Azzopardi Ernest, Joerg, Graf, Kon, Moshe, C, Lineaweaver William. "Preventing infective complications following leech therapy: Is practice keeping pace with current research?" Microsurgery. 2009;29(8):619-625.

89. Aravena-Román, Max, Timothy J. J. Inglis, Barbara Henderson, Thomas V. Riley, Chang Barbara J. Antimicrobial Susceptibilities of Aeromonas Strains Isolated from Clinical and Environmental Sources to 26 Antimicrobial Agents. Antimicrob Agents Chemother. 2012;56(2):11101112 .

90. Yun Luo, Julian Ye, Dazhi Jin, et al. Molecular analysis of non-O1/nonO139 Vibrio cholera isolated from hospitalized patients in China. BMC Microbiol. 2013;13(52):1-12. 
91. Quansah E, Barnie PA, Acheampong DO, et al. Geographical distribution of $\beta$-lactam resistance among Klebsiella spp. From selected health facilities in Ghana. Trop Med Infect Dis. 2019;4(3):1-14. doi:10.3390/tropicalmed4030117

92. Leila Soufi, Yolanda Sáenz, María de Toro, et al. Phenotypic and Genotypic Characterization of Salmonella enterica Recovered from Poultry Meat in Tunisia and Identification of New Genetic Traits. Vector-Borne Zoonotic Dis. 2012;12(1):10-16.

93. Fadlalla Imad M.T, Mohamed E. Hamid, Ahmed G. Abdel Rahim, Ibrahim, Mohamed T. Antimicrobial susceptibility of Salmonella serotypes isolated from human and animals in Sudan. J Public Heal Epidemiol. 2012;4(1):19-23

94. Senthilkumar B, Prabakaran G. Multidrug Resistant Salmonella typhi in Asymptomatic Typhoid Carriers among Food Handlers in Namakkal District, Tamil Nadu. 2005. 2005;23:92-94.

95. Yang H, Chen G, Zhu Y, et al. Surveillance of antimicrobial susceptibility patterns among Shigella species isolated in China during the 7-year period of 2005-2011. Ann Lab Med. 2013;33(2):111-115.

96. Yismaw Gizachew, Challa Negeri, Kassu Afework. A five-year antimicrobial resistance pattern observed in Shigella species isolated from stool samples in Gondar University Hospital, northwest Ethiopia. Ethiop JHealth Dev. 2006;20(3):194-198.

97. Ranjbar R, Aleo A, Giammanco GM, Dionisi AM, Sadeghifard N, C., Mammina. Genetic relatedness among isolates of Shigella sonnei carrying class 2 integrons in Tehran, Iran, 2002 - 2003. BMC Infect Dis. 2007;22:62.

98. Nabi Jomezadeh, Shahram Babamoradi, Enayatollah Kalantar and HJ. Isolation and antibiotic susceptibility of Shigella species from stool samples among hospitalized children in Abadan, Iran Gastroenterol Hepatol Bed Bench. Autumn. 2014;7(4):218-223.

99. Fulla Noelia, Valeria Prado, Claudia Dura, N, Rosanna Lagos, Levine Myron M. Surveillance for antimicrobial resistance profiles among Shigella species isolated from a semi-rural community in the Northern administrative area of Santiago, Chile. Am J Trop Med Hyg. 2005;72(6):851-854

100. Pazhani GP, T, Ramamurthy, U.Mitra, S. K. Bhattacharya, Niyogi K. Species diversity and antimicrobial resistance of Shigella spp. isolated between 2001 and 2004 from hospitalized children with diarrhoea in
Kolkata (Calcutta), India. Epidemiol Infect. 2005;133:1089-1095. 101. Barari, Savadkoohi Rahim, Ahmadpour-Kacho Mousa. Prevalence of Shigella species and their antimicrobial resistance patterns at Amirkola Children's Hospital, north of Iran. Iran J Pediatr. 2007;17(2):117-122.

102. Reema Nath, Lahari Saikia, Choudhury Gargi, Sharma Daisy. Drug resistant Shigella flexneri in \& around Dibrugarh, north-east India. Indian J Med Res. 2013;137(1):183-186.

103. Ruslan MS, Bektemirov AM, Ibadova GA, et al. Antimicrobial resistance patterns and prevalence of class 1 and 2 integrons in Shigella flexneri and Shigella sonnei isolated in Uzbekistan. Gut Pathog. 2010;2(1):18.

104. Timothy L Overman, Michael Janda J. Antimicrobial Susceptibility Patterns of Aeromonas jandaei, A. schubertii, A. trota, and A. veronii Biotype veronii. J Clin Microbiol. 1999;37(3):706-708.

\section{Peer Reviewed}

Competing Interests: None declared.

Received: 24 Jul 2019; Accepted: 27 Mar 2020.

Cite this article as: Olipher M, Muyodi J, Muthami A, Malela J, Mokaya T, Matilu M. Antibacterial Spectrum and Susceptibility of Bacterial Pathogens Causing Diarrheal Illnesses: Cross Sectional Study of Patients Visiting Health Facility in Lake Victoria Region - Kenya. E Afr Sci. 2020;1(2):45-58 http://doi.org/10.24248/EASci-D-18-00013

(C) Olipher et al. This is an open-access article distributed under the terms of the Creative Commons Attribution License, which permits unrestricted use, distribution, and reproduction in any medium, provided the original author and source are properly cited. To view a copy of the license, visit http://creativecommons.org/licens- es/by/4.0/. When linking to this article, please use the following permanent link: http://doi.org/10.24248/EASci-D-18-00013 